\title{
Lecciones de peruanidad a través del conocimiento de las fuentes
}

\author{
Nelly Mac Kee de Maurial \\ Unidad de Post-Grado
}

El doctor Jorge Basadre selló con una impronta de peruanidad la Escuela Nacional de Bibliotecarios, al fundarla en 1943. Esto se traduce en la labor docente que realizaron todos sus profesores, no obstante, consideramos que esta forja de peruanidad se manifiesta con caracteres especiales en tres maestros ya fallecidos, el doctor Alberto Tauro del Pino, don Ricardo Arbulú Vargas y don Alejandro Lostaunau Ulloa. De ellos nos ocupamos en este artículo.

\section{ALBERTO TAURO DEL PINO}

Puede afirmarse que ell lema del doctor Alberto Tauro del Pino, gran humanista, fue "peruano soy y nada de lo que atañe al Perú me es ajeno". La mejor prueba de esta afirmación está en su Enciclopedia ilustrada del Perú, cuya segunda edición se publicó en sếs tomós y constituye uno de sus principales legados. $\mathrm{Sin}$ - embarge, su-ediciôn ampliada y actualizada continúa inédita.

Justamente en esa obra monumental se preocupó por consignar gran parte de su rica producción hasta 1987, y en ella nos basamos para referirnos a la vastedad de su obra, además de las informaciones recibidas de su hija Talia.

El doctor Alberto Tauro nació en el Callao en 1914, hijo de Miguel Angel Tauro y Catalina del Pino. Estudió en el colegio de "La Inmaculada" y en la Universidad Nacional Mayor de San Marcos, graduándose en la Facultad de Letras con las tesis Mocedad de José Rufino Echenique (bachiller, 1939) y Presencia y definición del indigenismo literario (doctor, 1940). Realizó además los estudios completos de Derecho.

LETRAS (Lima), 94: 37-48,1997. 


\section{Docencia}

Un interesante como valioso aspecto de su vida profesional fue el dedicado a la docencia en colegios nacionales, desde 1933 hasta 1959; en el nivel superior en el Instituto Pedagógico Nacional de Varones que se convirtió en la Escuela Normal Superior (1949-1961), y en la Universidad Nacional Mayor de San Marcos desde 1945, donde se inició como catedrático en la especialidad de Historia. Desempeño cargos directivos en la misma universidad, en el Departamento de Publicaciones (1964-69), el Programa Académico de Ciencias Histórico-Sociales (1969-1970). Se encargó también de la Coordinación Académica y Evaluación Pedagógica (19731977) en la misma universidad y en una u otra forma se mantuvo vinculado a San Marcos hasta el final de sus días.

En la Escuela Nacional de Bibliotecarios, fue responsable de la formación de los futuros bibliotecarios, a través de la enseñanza de los cursos de Bibliografía Peruana, en donde inculcaba no sólo profundos conocimientos sino también el amor a las fuentes de la cultura peruana, que él poseía (1944-1958).

\section{Biblioteca Nacional}

Una faceta de isu vidatque le deparó grandes satisfacciones, según su hija Talia, fueron sus años dequabajoen la BibliotecaNacional, donde se inició, antes del incendio, como jefe de catalogación (1941-1943) y donde participó en la etapa de reconstrucción como jefe del Departamento de Ingresos (19431945), con el doctor Jorge Basadre y como jefe del Departamento de Investigaciones bibliográficas (1946-1959), con el doctor Cristóbal de Losada y Puga. Asimismo desempeño en forma interna el cargo de Director de la Biblioteca Nacional en cuatro oportunidades. Su paso por la Biblioteca Nacional fue fructífero no sólo porque allí gestó parte de su obra, sino también por su labor docente en la Escuela Nacional de Bibliotecarios.

\section{Comisiones honoríficas}

Entre sus importantes cargos, integró, en representación de la Universidad peruana la Comisión Nacional del Sesquicentenario de la Independencia. Participó en la Colección documental, que se publicó para este evento, preparando siete volúmenes, además de los siete que forman la 
edición princeps de la Historia del Protectorado de Germán Leguía y Martínez.

Fue miembro de la Academia Nacional de Historia, desde 1958; de la Sociedad Peruana de Historia, desde 1948; de la Academia Peruana de la Lengua desde 1980; del Centro de Estudios Históricos-Militares, de la Sociedad Geográfica de Lima y de la Sociedad Bolivariana del Perú, así como presidente de la Asociación Nacional de Escritores de Artistas en 1982.

\section{Editor}

Sus labores de editor se inician con la revista Prometeo (1930-1931). Fundó las revistas Palabra con José Alvarado Sánchez, Ernesto Gastelumendi Velarde y Augusto Tamayo Vargas (1936 a 1937 y 1944); Garcilaso, bajo el patrocinio de la Asociación Nacional de Escritores y Artistas; editó Biblión en 1942, Fénix, revista de la Biblioteca Nacional, números 5-12 (1947-1958); Boletín de la Biblioteca Nacional, números 10-20 (1947 a 1958).

El doctor Tauro del Pino rescató textos desconocidos o inéditos de diversos autores como Mariano Amézaga, Ramón Castilla, Luis Benjamín Cisneros, José Santos Chocano, Agustín Gamarra, Benito Laso, José Antonio de Lavalle, Francisisdo Xayier de Luna Pizarro,José Carlos Mariátegui, Domingo Martínez Luján, José Toribio Medina, Adán Felipe Mejía, Ricardo Palma, Felipe Pardo y Aliaga, Hugo Pesce, José María Quimper, Manuel Ascencio Segura, José Simeón Tejada, Manuel Lorenzo de Vidaurre y Francisco de Paula González Vigil.

\section{Periodismo}

Destacado periodista, colaboró en Jornada y La Prensa, obteniendo el Premio Nacional de Periodismo otorgado por el Ministerio de Educación Pública, en 1945. Publicó artículos diversos en periódicos y revistas nacionales y extranjeros.

\section{Contribución a la bibliografia peruana}

La labor del doctor Tauro como bibliógrafo es la más importante realizada en el siglo XX. Publicó en este aspecto Contemporáneos y Cultu- 
ra, dos revistas de la generación modernista (1938); El Espejo de mi Tierra (1942); Anuario Bibliográfico Peruano de 1945 a 1959, correspondientes a la producción de 1945 a 1959, Bibliografía peruana de historia, 19401953 con un suplemento en 1958, Bibliografía peruana de legislación y estudios jurídicos, 1943-1954; Guia de estudios históricos (1955) Bibliografía peruana de literatura 1931-1958; Amauta y su influencia (1960); Felipe Pardo y Aliaga periodista (1962); Hacia un catálogo de seudónimos peruanos (1965); y Bibliografía del Inca Garcilaso de la Vega, 1966.

Publicó antologías literarias e históricas: Poesía de la Historia del Perú (1948), Navidad en la literatura peruana (1948), Imagen del Perú (1960), Viajeros en el Perú republicano (1967), La Independencia Nacional y las políticas de las potencias (1969) y con Félix Denegri Luna y Armando Nieto Vélez, Antología de la independencia del Perú (1972).

Estudios literarios

Los estudios literarios del autor que él mismo encuentra más destacados aún El indigenismo a través de la poesia de Alejandro Peralta (1935), Presencia y definición del indigenismo literario (1940), Esquividad y gloria de la Academia Antártica (1948), Clorinda Matto de Turner y la novela indigenista (1976).

Textos históricos y biggráficos ccinelli Converso"

\section{Biblioteca de Letras}

Historia e historiadores del Perú, 1943-1946 (1949), La fundación de la Biblioteca Nacional (1951), Crónicas del Japón (1960), Crónicas de Filipinas (1961), Manuel de Odriozola, prócer, erudito, bibliotecario (1964), Diccionario enciclopédico del Perú(1966), 3 vols., la 1a. ed. En 1987 Peisa publicó una segunda edición corregida y aumentada, en 6 vols. (1987), Perú época republicana, t. 1, (1973), Rectores de la Universidad Nacional Mayor de San Marcos, siglo XIX (1978 y 1980) y la Defensa de Lima (1982).

Mariateguismo

Mención especial merece su acendrada devoción por la obra de José Carlos Mariátegui a cuyo estudio se dedicó desde la juventud. En la revista Palabra publicó con una introducción, 23 años de sucesos extranjeros de José Carlos Mariátegui. Revisó los artículos de la revista Amauta y editó en 
1960 Amauta y su influencia, primera presentación sistemática e índices de la revista, antes de que se realizara la edición facsimilar. Copió mecanográficamente los artículos de Mariátegui dispersos en revistas peruanas y extranjeras para introducirlos en las ediciones del Amauta que sus hijos realizaron. Participó especialmente en la compilación del Alma matinal y otras estaciones del hombre de hoy con notas introductorias. Escribió también la introducción a La novela y la vida (1955) y El artista y la época, a la edición facsimilar de Amauta, Labor (1974) y Nuestra época (1985). Fue nombrado Presidente honorario de la Comisión Nacional del Centenario de José Carlos Mariátegui. Trabajó con los hijos de Mariátegui, que lo consideraban un hermano mayor, en la publicación del Anuario Mariateguiano, que dirigió con Antonio Melis desde 1989. Seleccionó los textos de siete, de los ocho volúmenes, que conforman la edición de Escritos juveniles de José Carlos Mariátegui (1987-1993), que comprenden los artículos publicados por Mariátegui con el seudónimo de Juan Croniqueur, la que publica con textos introductorios.

\section{Ediciones póstumas}

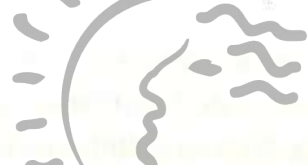

Luego de su muerte aparecièron algunas ediciones póstumas como su Catálogo de seudónimos peruanos, edición ampliada; Quinientos años de modernidad, separata del libro II Congresö de Academials Ibēroamericanas de la Historia, publicado por la Real Academia de la Historia de España: Tradiciones peruanas de Eleazar Boloña, compilación y prólogo de Alberto Tauro; y Teoría e investigación del folklore con prólogo de Alberto Tauro, editado por la Universidad Nacional Mayor de San Marcos, en donde se encarga del capítulo referente a Antonio Ricardo.

\section{Inéditos}

Dejó inéditos: Biografía, epistolario y memorias de Andrés Avelino Cáceres; El catálogo de gentilicios; la edición ampliada de Contribuciones a la historia del periodismo; y la edición ampliada y actualizada de la Enciclopedia ilustrada del Perú.

El doctor Alberto Tauro del Pino falleció víctima de una repentina enfermedad el 18 de febrero de 1994, dejándonos el ejemplo de su infatigable capacidad de trabajo intelectual, de su versatilidad y de su gran amor y conocimiento del Perú cuyos frutos constituyen un importante don para todos los peruanos. 
Don Ricardo Arbulú Vargas consagró 21 años de su vida a la Biblioteca Nacional, con amor y entera dedicación; se entregó también a la difusión de la cultura puneña y cultivó una hermosa amistad con Martín Adán, quien afirmó que el "único hermano de padre y madre que yo he encontrado en el mundo es Ricardo Arbulú". Trabajo hasta el final de su vida en la biblioteca Pedro Benvenutto Murrieta, de la Universidad del Pacífico para cuyo procesamiento creó un esquema de clasificación.

Actuó como un orfebre de los registros bibliográficos sentando, con un equipo, las entradas de autoridad para el catálogo de autores personales e institucionales, peruanos y elaboró, coordinando con los bibliotecólogos de su entorno, la Tabla Perú, adaptación del sistema de clasificación de Melvil Dewey, para las necesidades de la bibliografía peruana.

Nació en Puno el 20 de noviembre de 1907, realizó sus estudios de primaria en el Colegio Nacional de San Carios de Puno y la secundaria en la Deutsche Schule de Lima. Siguió estudios de Filosofía e Historia en la Universidad Católica, donde se graduó con la tesis Proceso metafísico de la Independencia hispanoamericana (1937). Estudió Derecho en la Universidad Nacional Mayor do San Marcos.

Fue miembro de lá primera promoción de alumnos de la Escuela Nacional de Bibliotecarios que tuvo como profesores al grupo de "Amigos de la Biblioteca Nacional del Perú”, bibliotecólogos que vinieron de Estados Unidos después del incendio de nuestra Biblioteca Nacional. Su vocación por el trabajo bibliotecológico se había ya manifestado, pues, desde 1932, desempeñaba el cargo de bibliotecario del hospital "Víctor Larco Herrera", donde conoció a Martín Adán.

\section{Biblioteca Nacional}

En 1942 se inició como catalogador en la Biblioteca Nacional, acudiendo a un llamado, con un grupo de estudiantes universitarios que convocó el doctor Manuel Beltroy. Después del incendio, simultáneamente a los estudios de Bibliotecología, trabajo hasta altas horas de la noche con sus colegas de promoción, recuperando libros de los escombros, clasificando y catalogando 
los volúmenes de la colección peruana, en la Escuela Nacional de Bellas Artes, donde se trasladó la Biblioteca Nacional, hasta que se terminó el nuevo local que se construía en ese entonces.

Posteriormente fue nombrado jefe del Departamento de Clasificación y Catalogación y en los últimos años Coordinador de la Biblioteca Nacional, cargo que desempeñó hasta 1973. En el ejercicio de sus funciones impulsó la organización y registro de la colección documental de la Biblioteca Nacional, de acuerdo a las normas internacionales vigentes, fomentando la adaptación de las mismas a las peculiaridades de las fuentes peruanas.

Al dejar la Biblioteca Nacional ejerció el cargo de director de la Biblioteca Riva-Agüero de la Pontificia Universidad Católica y desde 1980 se encargó de organizar y dirigir la biblioteca Pedro Benvenutto Murrieta, de la Universidad del Pacífico.

Docencia y distinciones

Ejerció la docencia en la Escuela Nacional de Bibliotecarios, teniendo a su cargo los cursos de Clasificación y Técnica bibliográfica, curso éste para el que escribió el Prontuario de técnica bibliográfica (1952).

En la Escuela Académico Profesional de Bibliotecología y Ciencias de la Información de la Universidad Nacional Mayor de San Marcos dictó los cursos de Introducción a la Bibliotecología y Ciencias de la Información y el de Procedimiento de la información.

En el Centro de Instrucción Militar del Perú el curso de Introducción a la filosofía, de 1956 a 1968.

Fue delegado del Perú ante el Seminario de Bibliografía y Documentación Científica y Técnica que se realizó en México en 1960 y en la Conferencia Internacional de Catalogación efectuada en París, en 1961. En esos años asumió la presidencia de la Asociación Peruana de Bibliotecarios.

\section{Promoción de bibliotecas}

Indujo a la creación de la Biblioteca Popular "Marcos Fidel Suárez" 
en la urbanización El Agustino y se preocupó por la creación de una biblioteca regional puneña, encargo que hizo a José Luis Ayala Olazábal, a quien entregó 300 fichas bibliográficas para sentar las bases de su catálogo. Esta biblioteca recibió el nombre de don Ricardo Arbulú, lamentablemente fue destruida tiempo después, según informa el mismo Ayala Olazábal. Alentó también la organización y sistematización de la Biblioteca Municipal de Puno, a través de cursos de capacitación, para lo cual encargó la labor a expertas bibliotecólogas, sus discípulas, Erlinda Chávez y Zorayda Loyola.

\section{Puneñismo esencial}

Puso de manifiesto su amor a Puno tanto en la compilación de textos de la literatura regional puneña, que coleccionó y mostraba con orgullo, como en la fundación del Instituto Punneño de Cultura (1956), desde el que desarrolló gran actividad en pro de la difusión de los valores culturales puneños; $y$, en los trabajos de investigaciôn que realizó y que permanecen inéditos.

Nos ha dejado una importante obra Punofilia en Lima, cuyo texto está prácticamente listo para su impresión Consta de 4 capítulos, el último de los cuales ha sido incorporado por sus familiares y amigos. El título significa: "Amor hacia Pund en Lima". Eleprimer capítulo; Opúsculos circunstanciales compila escritos sobre:Puno desde_1,956 a 1993. El segundo Ante la apacheta de los amautas, reúne los discursos fúnebres pronunciados por él en las exequias de puneños notables como Federico More, Alejandro Peralta, Enrique Encinas, Pablo Iturri, Dante Nava, Emilio Romero, etc. El tercero Recensiones, Puno en los textos, esto es, prólogos de su pluma, a libros de puneños sobre Puno. El tomo cuarto acopia los discursos necrológicos que se pronunciaron en sus funerales y en los homenajes que se le hicieron posteriormente.

La Tabla de clasificación AB (Arbulú-Benvenutto), también inédita se preparó "en homenaje a Pedro Benvenutto Murrieta y para aplicarla a esta colección, en acuerdo mutuo con el donante, quien era, además de consumado bibliógrafo, un experto en el estudio, aplicación, crítica de los sistemas vigentes ( $D C, C D U, L C$, etc.), de modo que su orden temático estuviera conforme al proceso evolutivo de las varias ramas de la cultura humana, esto es en buena cuenta con la historia universal, según ese crite- 
rio histórico la colección estaría ordenada siguiendo las etapas sucesivas del desarrollo histórico de cada cual de sus sectores temáticos; los autores a su vez estarian subordinados según la respectiva época histórica durante la cual produjeron su obra escrita, en lugar de estudiarla según el orden establecido e históricamente inútil. Para simbolizar las divisiones de mayor jerarquía y extensión temática se ha elegido las primeras letras del alfabeto latino, luego se ha desarrollado en lo posible también históricamente los números digitos utilizándose en algunos casos el punto decimal: A Religión, B Mitología, C Arte, D Lingüística y filología, E Literatura, $F$ Filosofía, G Ciencia, H Técnica, J Ciencias Sociales, K Geografía, I y Ll Historia, $N$ Omnia, $R$ Referencias, $S$ Bibliografia, $T$ Hemeroteca, $X$ Documentatoteca".

Es autor además de documentos inéditos sobre la Biblioteca Nacional y la Escuela Nacional de Bibliotecarios que la familia y los amigos publicarán próximamente.

Sociedades

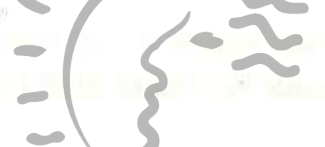

Fue miembro y secretario fundador del Instituto Peruano de Cultura Hispánica (1947-1979), de la Sociedad Peruana de Historia (1945-1978) y Presidente de la Conferenoiadeisañ Vicente de Pauldedos Sagrados Corazones.

\section{"Jorge Puccinelli Converso»}

Falleció el 25 de mayo de 1995, habiendo trabajado en la Biblioteca Pedro Benvenutto Murrieta, prácticamente hasta el final de sus días.

\section{Homenajes}

Recibió un homenaje en vida el 5 de julio de 1973 que le ofrecieron los bibliotecarios, intelectuales, amigos y discípulos "por haber sido elemento principal en la restauración y tecnificación de la Biblioteca Nacional, al término de su excepcional labor en 27 años consecutivos en dicha institución".

Los alumnos de la Escuela Académico Profesional de Bibliotecología y Ciencias de la Información de la promoción Pedro Zulen, de la Universidad Nacional Mayor de San Marcos, le rindieron un homenaje en la Facultad de Letras de dicha universidad. Una publicación conmemorativa, con 
cubierta de madera, realizada por Orlando Corzo y Sabine Lumbreras, de circulación restringida, fue distribuida entre los participantes.

Recibió homenajes póstumos asimismo de la Universidad del Pacífico y del Instituto Puneño de Cultura. Podemos decir él que fue un bibliotecólogo ejemplar, un puneño esencial y un peruanista a carta cabal.

\section{DON ALEJANDRO LOSTAUNAU ULLOA}

Autodidacta paradigmático, podemos decir de él que fue gran bibliotecario y bibliógrafo excepcional. Trabajó cuarenticinco años en la Biblioteca Nacional, y en esas cuatro y media décadas se consubstanció con la bibliografía peruana. Sus conocimientos fueron muy apreciados por los investigadores de la cultura peruana, para quienes constituía la mejor fuente de consulta, por encima de los catálogos y las bibliografías existentes.

Nació en Lima el 4.de diciembre de 1904 y falleció el 5 de octubre de 1992, habiéndose dedicado a la actividad intelectual hasta la postrera etapa de su existencia.

\section{Biblioteca Nacional}

\section{Biblioteca de Tetras}

Ingresó a trabajar en la Biblioteca Nacional, el, 22 de agosto de 1992, cuando era director el doctor Alejandro O. Deustua, de modo que conoció la vieja y la que se llamó nueva Biblioteca Nacional, organizada después del incendio de 1943, de acuerdo a los principios básicos de la flamante ciencia de la Bibliotecología. Se desempeño como jefe de la Sala Perú, que hasta ahora aloja la colección peruana y peruanista y que constituye el núcleo de la Biblioteca Nacional; colección que don Alejandro conoció profundamente.

\section{Docencia}

Colaboró el doctor Alberto Tauro del Pino en la cátedra de Bibliografía Peruana, en Escuela Nacional de Bibliotecarios, desde 1949 hasta 1958, y al retirarse el doctor Tauro, siguió encargándose de los talleres de Bibliografía Peruana. Colaboró asimismo con el doctor Fernando Silva Santisteban, la doctora Josefina Ramos de Cox, el padre Rubén Vargas Ugarte, entre otros, hasta 1967. Fue don Alejandro para 
sus alumnos el más entusiasta para el conocimiento de las fuentes de la cultura patria.

Instituto Riva-Agüero

Organizó y dirigió la biblioteca del Instituto Riva-Agüero de la Pontificia Universidad Católica del Perú, hasta la década del ochenta.

\section{Publicaciones}

Escribió diversos artículos de su especialidad, en los que fue plasmando sus conocimientos, sin embargo no nos dejó la obra integral que como bibliógrafo pudo haber preparado.

Es autor de El desconocido manuscrito de Pereyra y Ruiz sobre A requipa (1946), Luis Jerónimo de Oré: Descripción, sitio, tierra y población del Perú (1951), Enrique Torres Saldamando, historiador y bibliógrafo olvidado (1956), Dos ediciones de la crónica del Perú de Pedro Cieza de León (1959), Biobibliografía de Manuel Vicente Villarán (1958), Diego Fernández (El Palentino), (1963), Indice por materias y auivies de los XXV volúmenes de la Revista Histórica del Perú (1964), Biobibliografía del reverendo padre Rubén Vargas Ugarte (1967), Belaunde y su aporte bibliográfico a la emancipación de América (1969), Biobibliografía de Carlos Salazar Romero (1971), 56 proclamas de curas en la emancipación (1971), El cronista Agustín de Zárate (1972), Bibliografía republicana, consideraciones en torno a su estudio (1974), Aspectos bibliográficos sobre el conflicto de 1879; los limites con nuestros vecinos, conflictos limítrofes; los limites por el sur; aspectos económicos; sobre el guano; obras generales; sobre la campaña del sur; estudios biográficos; publicaciones periódicas, fuentes bolivianas y chilenas, (1979).

Escribió también Domingo Nieto, el mariscal más joven que tuvo el Perú (1980); Arica: lucharemos hasta quemar el último cartucho (1980) y Los héroes del morro de Arica (1980).

Como se advierte su valiosa obra revela un profundo amor al Perú, Alberto Tauro, Ricardo Arbulú Vargas y Alejandro Lostaunau Ulloa, con su amor por la patria, nos han dejado las más valiosas lecciones de peruanidad. 


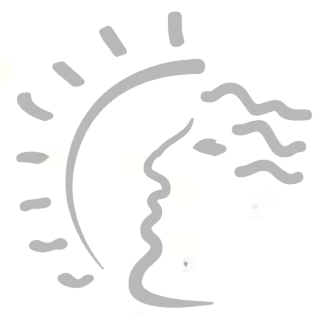

Biblioteca de Letras "Jorge Puccinelli Converso" 